\title{
COSMOLOGICAL SIZE EVOLUTION OF EXTRAGALACTIC
}

\section{RADIO SOURCES}

\author{
ASHOK K. SINGAL \\ Physical Research Laboratory \\ Astronomy and Astrophysics Division \\ Navrangpura, Ahmedabad - 380 009, India. \\ E-mail: asingal@prl.ernet.in
}

The study of cosmological evolution of the sizes of extragalactic radio sources started about a quarter century back. From the very first angular size-redshift $(\theta-z)$ plots (Miley 1968, 1971; Legg 1970) and angular sizeflux density $(\theta-S)$ plots (Swarup 1975; Kapahi 1975) it became evident that some sort of cosmic epoch-dependent evolution in the size distribution for the population of extragalactic radio source needs to be proposed; the sources at earlier epochs appeared on the average to have smaller physical sizes. However, a suitable luminosity-linear size $(P-l)$ correlation among the radio source population could also explain the observations, without invoking a size evolution with redshift. The only reliable way to disentangle these two separate effects is to investigate the size distribution in the luminosity-redshift plane, where one could examine not only the $l-z$ relation for a given luminosity class, but could also check for a $P$ - $l$ correlation in a given redshift bin.

Such an approach was followed by Oort et al. (1987); Singal (1988); Kapahi (1989), who concluded that the physical sizes of powerful radio galaxies (PRGs) appear to evolve rapidly with redshift $\left(l \propto(1+z)^{-3}\right)$. In addition it was also pointed out that there is a direct correlation between luminosity and size among PRGs, the more luminous ones are larger in radio sizes. At the same time Singal (1988) cautioned that, unlike in PRGs, quasars do not show a direct luminosity-size correlation. On the contrary, there is a hint of an inverse correlation; the more luminous quasars appear to have smaller physical sizes. Moreover, the size evolution of quasars, if any, appears to be much weaker (see also Barthel and Miley 1988). Later Singal (1993), using a larger sample of sources that included many more galaxies at high redshifts, found the difference between the two distributions 


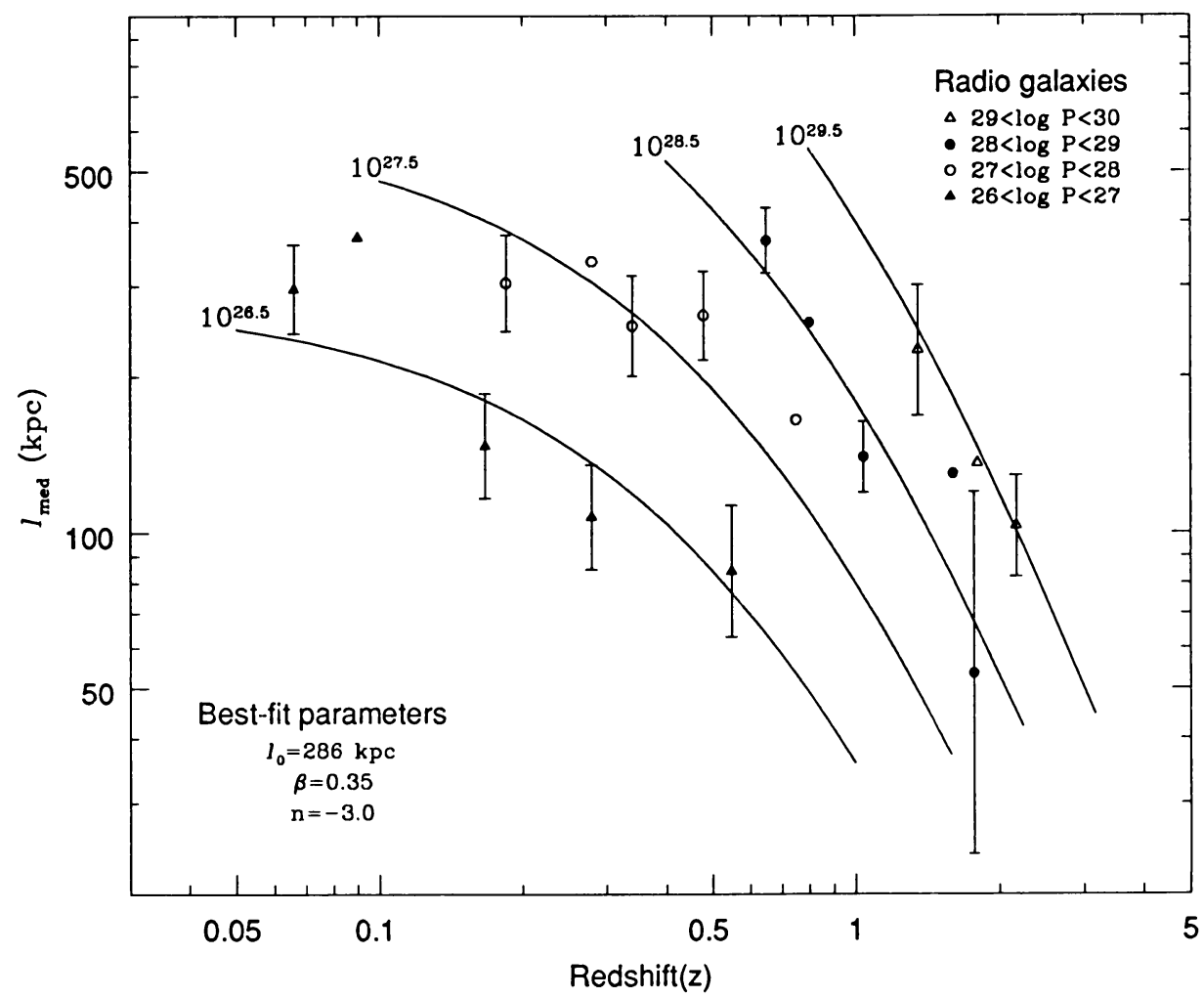

Figure 1. The change in $l_{m e d}$ with redshift for radio galaxies in different luminosity bins. The plotted points with error bars are from Singal (1993), while those without error bars represent the $3 \mathrm{C}$ and $6 \mathrm{C}$ data of Neeser et al. (1995). The family of curves is drawn according to the relation $l=l_{0}\left(P / 10^{26.5}\right)^{\beta}(1+z)^{n}$, for the best-fit parameter values $l_{0}=286 \mathrm{kpc}, \beta=0.35, n=-3$ (Singal 1993).

to be even more statistically significant. This difference in fact, provides a strong evidence against the simple orientation-based unified scheme models (Barthel 1989).

However, there are other publications in the literature with conclusions contrary to the above. For example, Nilsson et al. (1993) have claimed that in their studies they find no significant differences between the radio sizes of quasars and PRGs. They have reported the presence of a negative correlation between radio size and power among PRGs, a result in contradiction with almost all other previous studies. Moreover, they find no need for a cosmic evolution of radio source size. On the other hand, Neeser et al. (1995), from their sample containing $3 \mathrm{C}$ and $6 \mathrm{C}$ sources, have claimed that there is no evidence of a $P-l$ correlation among PRGs and that a milder size evolution $\left(\left(l \propto(1+z)^{-1.5}\right)\right.$ may be present.

This is a highly confusing situation. Perhaps the first step in resolving 


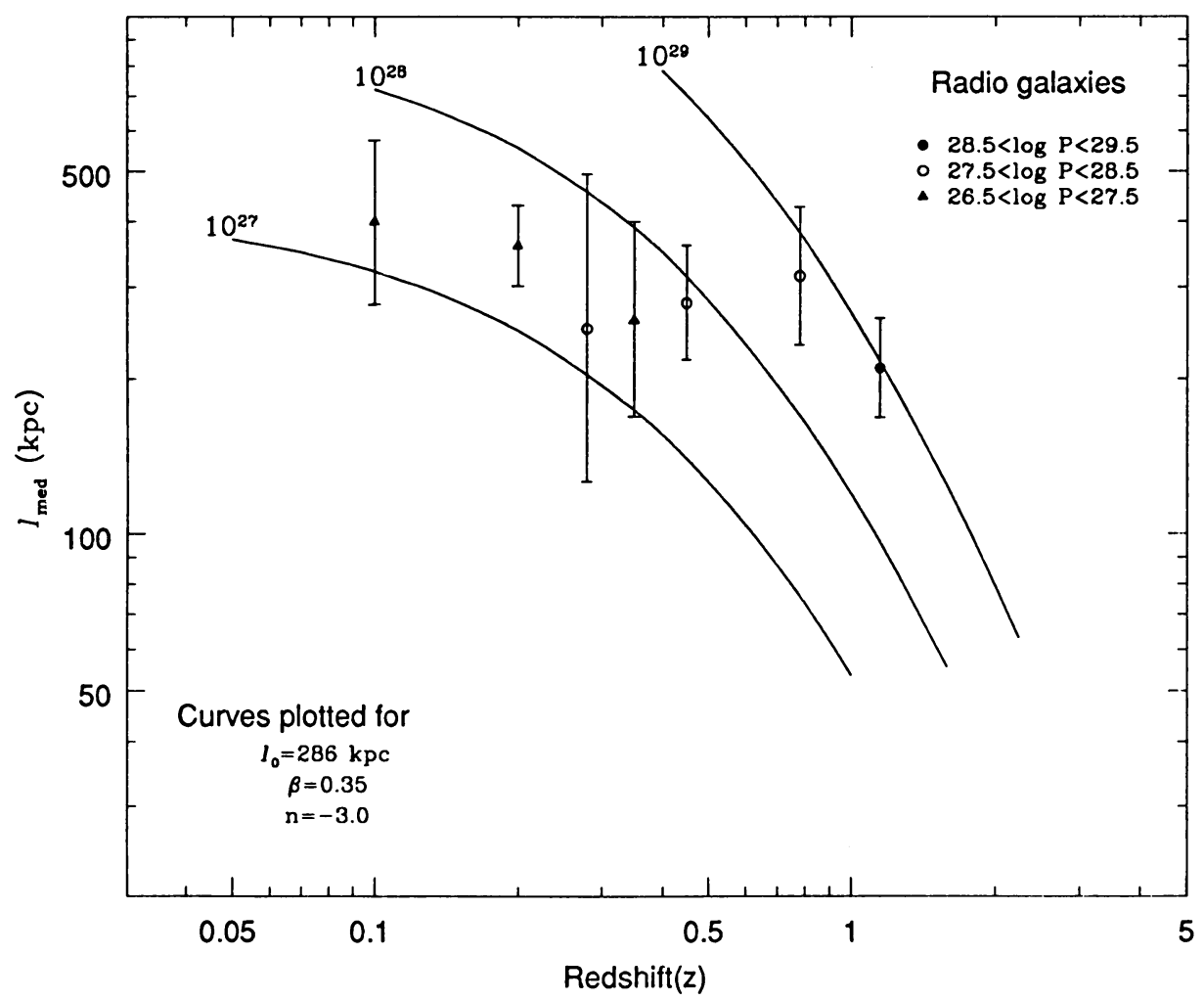

Figure 2. A plot of $l_{\text {med }}$ with redshift for the radio galaxies sample from Nilsson et al. (1993). The family of curves is drawn according to the relation $l=l_{0}\left(P / 10^{26.5}\right)^{\beta}(1+z)^{n}$, for the parameter values $l_{0}=286 \mathrm{kpc}, \beta=0.35, n=-3$ derived by Singal (1993).

these discrepancies would be to check whether the differences lie in the data used in various studies, or are these caused merely by the different statistical methods used by different authors. According to Neeser et al. (1995), the main reason for different estimates does not seem to lie in the different statistical approaches used, and that the differences may lie in the radio size data. We decided to check this here by plotting the median size values estimated from the data used by Nilsson et al. (1993) and Neeser et al. (1995) on Singal's (1993) $P-z-l$ diagram, and to ascertain wherein the discrepancies might lie.

Fig. 1 shows the $P$ - $z$ - $l$ diagram from Singal (1993), depicting the change in $l_{\text {med }}$ with redshift for radio galaxies in different luminosity bins. On this diagram we have also plotted $l_{m e d}$ values in different $P-z$ bins for the sample used by Neeser et al. (1995). Unfortunately it was not possible to give reliable error estimates for these points (especially for the $6 \mathrm{C}$ points, mainly because at the moment their tabulated data is not available to us). However, one thing is clear. There is not much discrepancy in the size 
values in the two data sets. The $l_{\text {med }}$ values from Neeser et al. (1995) appear to be quite consistent with the curves drawn using the best-fit parameter estimates from Singal (1993). It also appears that from the 3C and 6C data alone one will be left with a wide range of $\beta$ and $n$ values, mainly because of a small coverage of the $P-z$ plane.

In Fig. 2 we have plotted the $l_{m e d}$ values estimated from Nilsson et al. (1993). We have retained the luminosity bins above the FR II limit only. For a comparison we have also plotted the family of curves drawn for the parameter values derived by Singal (1993). There are no significant deviations from the drawn curves. However the plotted points do not, in themselves, put a heavy constraint on the range of $\beta$ and $n$, mainly because of the lack of sufficient data especially at higher redshifts in various luminosity bins.

It appears that the differences in results, arrived at by different authors, have arisen mainly because these are based on samples which may be lacking sufficient data in various $P-z$ bins. It appears that the situation is likely to improve only with the availability of larger samples covering larger range in the $P-z$ plane. Samples, of course, have to be selected at metre-wavelengths and with sufficient care so that no radio-size based bias sneaks into them.

In the last IAU Symposium on Extragalactic Radio Sources, Baldwin (1982) had contemplated on the possibility of finding evolutionary tracks of radio sources in the $P-D$ ( $P-l$ in the present notation) diagram, the hope was that one may be able to find something akin to an H-R diagram for radio sources. However, the difficulties involved in the case of extragalactic radio sources appear to be of a 'higher dimension'. Because of the cosmological size evolution of these sources we need to consider a 3-dimensional $(P-l-z)$ version of such a diagram if we want to get a hold on the evolutionary tracks of the radio sources.

\section{References}

Baldwin, J. E., 1982, in Extragalactic Radio Sources, IAU Symp. 97, eds. Heeschen, D. S., Wade C. M. , Reidel, Dordrecht, p. 21

Barthel, P. D., 1989, ApJ, 336, 606

Barthel, P. D., Miley, G. K., 1988, Nature, 333, 319

Kapahi V. K., 1975, MNRAS, 172, 513

Kapahi, V. K., 1989, AJ, 97,1

Legg, T. H., 1970, Nature, 226, 65

Miley, G. K., 1968, Nature, 218, 933

Miley, G. K., 1971, MNRAS, 152, 477

Neeser, M. J., Eales, S. A., Law-Green, J. D., Leahy, J. P., Rawlings, S., 1995, ApJ, 451, 76

Nilsson, K., Valtonen M. J., Kotilainen J., Jaakkola T., 1993, ApJ, 413, 453

Oort, M. J. A., Katgert, P., Windhorst, R. A., 1987, Nature, 328, 500

Singal, A. K., 1988, MNRAS, 233, 87

Singal, A. K., 1993, MNRAS, 263, 139

Swarup, G., 1975, MNRAS, 172, 501 